\title{
Evaluation of Hydrogen Absorption into Iron by Alternating Current Responses in an Electrochemical Hydrogen Permeation Cell
}

\author{
Eiji TADA* and Atsushi NISHIKATA \\ Department of Chemistry and Materials Science, Graduate School of Science and Engineering, Tokyo Institute of Technology, \\ 2-12-1 Ookayama, Meguro-ku, Tokyo, 152-8552 Japan.
}

(Received on July 13, 2015; accepted on August 10, 2015; J-STAGE Advance published date: February $25,2016)$

\begin{abstract}
In this paper, hydrogen absorption behavior into iron was investigated by measuring alternating current responses for hydrogen-entry side and hydrogen-withdrawal side in an electrochemical hydrogen permeation cell. Hydrogen absorption efficiency of iron was evaluated as a function of electrode potentials by using both DC and AC components of the current responses. The hydrogen absorption efficiency of iron increased with decreasing cathodic polarization. Furthermore, frequency response characteristics of the hydrogen absorption efficiency were investigated at various polarization potentials and the results indicated that the hydrogen absorption efficiency decreased with increasing frequency of the perturbation potential. The frequency response characteristics of the hydrogen absorption efficiency was also simulated by using Fick's second law and the results suggested that hydrogen absorption kinetics can be considered to be faster than diffusion of hydrogen atoms in iron and to be under an equilibrium condition. By analyzing AC responses for hydrogen-entry side and hydrogen-withdrawal side, hydrogen absorption behavior into iron can be successfully evaluated.
\end{abstract}

KEY WORDS: electrochemical hydrogen permeation test; Devanathan-Stachurski permeation technique; potential perturbation; frequency response characteristics; electrochemical impedance.

\section{Introduction}

Environmental degradation of steels due to hydrogen embrittlement has been a great concern with increasing their strength because the susceptibility of steels to hydrogen embrittlement is highly relevant with the strength of the steels. When steels are used in atmospheric environments, aqueous corrosion of the steels can take place under a thin water layer formed by rainfall and/or dew. At this time, on the surface of the steels, hydrogen evolution reaction can be evolved as one of the cathodic reactions. According to the reports by some researchers, ${ }^{1-5)}$ in atmospheric corrosion, $\mathrm{H}$ atoms can be absorbed into steels during the corrosion in the atmospheric environments. For example, if the concentration of absorbed $\mathrm{H}$ atoms reaches in the order of sub ppm, $1000 \mathrm{MPa}$-class steels may be susceptible to hydrogen embrittlement. ${ }^{6}$ ) Therefore, it is quite important to clarify the mechanism on hydrogen absorption into steels during corrosion in atmospheric environments and to evaluate how much amount of $\mathrm{H}$ atoms can be absorbed into steels during the corrosion.

As mentioned above, during aqueous corrosion of steels, hydrogen evolution reaction (HER) can take place as a

\footnotetext{
* Corresponding author: E-mail: tada.e.aa@m.titech.ac.jp DOI: http://dx.doi.org/10.2355/isijinternational.ISIJINT-2015-414
}

cathodic reaction, because corrosion potential of steels in neutral aqueous media is less noble than the equilibrium potential of $\mathrm{H}^{+} / \mathrm{H}_{2}$. The reaction scheme of HER on a metal has been proposed to be a multistep as follows. ${ }^{7,8)}$

$$
\begin{gathered}
\mathrm{H}^{+}+\mathrm{e}^{-} \longrightarrow \mathrm{H}_{\mathrm{ad}} \ldots . \\
\mathrm{H}_{\mathrm{ad}}+\mathrm{H}_{\mathrm{ad}} \longrightarrow \mathrm{H}_{2} \ldots \\
\mathrm{H}^{+}+\mathrm{H}_{\mathrm{ad}}+\mathrm{e}^{-} \longrightarrow \mathrm{H}_{2}
\end{gathered}
$$

The first step (Eq. (1)) is Volmer reaction. This is an electrochemical reaction to form atomic $\mathrm{H}$ adsorbed on a metal surface. Some of the adsorbed $\mathrm{H}$ atoms can be combined with each other, forming molecular hydrogen. This is called Tafel reaction (Eq. (2)) and it is just a chemical reaction. By contrast, the adsorbed $\mathrm{H}$ atoms formed by Eq. (1) can become molecular hydrogen electrochemically by a different step in Eq. (3). This is so-called Heyrovsky reaction. Therefore, the formation of hydrogen molecules for HER on a metal begins with Volmer reaction followed by either Tafel reaction or Heyrovsky reaction depending on kinds of metals or surface conditions and so on. ${ }^{9)}$

By contrast, a part of the $\mathrm{H}$ atoms formed on a steel surface can be absorbed into the steel. Then the absorbed $\mathrm{H}$ atoms can diffuse into the steel and interact with defects or traps in it and then hydrogen embrittlement may be induced. 
Therefore, we think that in order to look for a way to mitigate the occurrence of hydrogen embrittlement of steel, the detailed mechanism of hydrogen absorption reaction (HAR) and the amount of $\mathrm{H}$ atoms absorbed into the steel should be clarified. To do that, we are focusing on hydrogen absorption efficiency of steel, ${ }^{10)}$ which is how much amount of $\mathrm{H}$ atoms can be absorbed into a steel, and on the kinetics of HAR into the steel, which means how fast the absorption reaction of $\mathrm{H}$ atoms can take place.

In order to investigate hydrogen absorption behavior into steels, so-called Devanathan-Stachurski permeation technique has been widely used. ${ }^{11-14)}$ This technique is an electrochemical technique and can evaluate in-situ the amount of absorbed $\mathrm{H}$ atoms from the surface of a metallic material by the measurement of oxidation current of the $\mathrm{H}$ atoms diffusing through the material at the opposite surface. From the results obtained by this technique, diffusion coefficient of $\mathrm{H}$ atoms in the material and surface concentration of the adsorbed $\mathrm{H}$ atoms can be evaluated. Furthermore, hydrogen absorption efficiency can be estimated by comparison of the oxidation current of $\mathrm{H}$ atoms with hydrogen evolution current. Since the occurrence of hydrogen embrittlement of steels can be associated with the amount of the absorbed hydrogen, it is quite important to investigate the hydrogen absorption efficiency of steels as a function of potentials. In addition, hydrogen absorption kinetics at the very surface of steels should be a critical information to find a way of preventing the steels from absorption of $\mathrm{H}$ atoms.

In this study, hydrogen absorption behavior into iron is evaluated using electrochemical hydrogen permeation technique. Furthermore, frequency response characteristics of hydrogen absorption efficiency into iron are measured using AC responses with an electrochemical hydrogen permeation technique, aiming at investigate the mechanism of hydrogen absorption reaction into iron.

\section{Experimental}

\subsection{Sample}

The material used in this study was an iron bar (Fe-0.006 mass $\%$ C-0.01 mass\%Si-0.021 mass\%Mn) of ca. $45 \mathrm{~mm}$ in a diameter. The iron bar was fully annealed at $850^{\circ} \mathrm{C}$ for 2 hours in a furnace. After the heat treatment, the iron bar was machined into a thin disk of $40 \mathrm{~mm}$ in a diameter and $0.5 \mathrm{~mm}$ in thickness. The both sides of the iron disk were abraded with SiC papers successively down to P1200 grit and were subjected to chemical etching in a commercial acidic bath containing hydrogen peroxide and fluoride ions (CPL-100, Ryoko Chemical Co., Ltd., Japan). After being taken out from the etching bath, the iron disk was immersed into an alkaline solution for neutralization, rinsed with Milli-Q water $(18 \mathrm{M} \Omega \mathrm{cm})$ and then blown with dry air. Finally, the thickness of the iron disk becomes roughly $0.45 \mathrm{~mm}$, although the exact thickness of the iron disks was measured before an experiment. A Ni wire for electric surfaces of the disk, electrodeposition of Pd was performed in a bath (K-pure Pd, Kojima Chemicals Co., Ltd., Japan) at a constant current density of $-15 \mathrm{~mA} / \mathrm{cm}^{2}$, aiming to get a $400 \mathrm{~nm}$ coating on the surface. Figure 1 shows a schematic drawing of an iron-disk sample prepared by the above procedure.

\subsection{Electrochemical Set-up}

In this study, hydrogen permeation current was measured by a Devanathan-Staturski permeation technique. ${ }^{11-14)}$ This technique has been applied to the measurement of hydrogen absorption into metallic materials and confirmed to be quite useful for in-situ evaluation of the amount of hydrogen absorption into metals and alloys. ${ }^{15-17)}$

Normally, in the Devanathan-Stachurski permeation technique, two identical vessels in each of which electrochemical measurements can be performed with a conventional threeelectrode-system are prepared, and then a thin metallic film, "membrane", is placed between the vessels and tightened up with them. In this study, two identical glass vessels were used; one of the glass vessels used for the hydrogen-entry side is referred to "H-entry-side" cell, and the other is for the hydrogen-withdrawal side to "H-withdrawal-side" cell, as shown in Fig. 2. A sample of an iron disk with Pd coating was set tightly between $\mathrm{H}$-entry-side and $\mathrm{H}$-withdrawal-side cells. At this time, the Pd-coated side of the sample was faced to the H-withdrawal-side cell.

In H-entry-side cell, an aqueous solution of $0.5 \mathrm{kmol} / \mathrm{m}^{3}$ $\mathrm{NaCl}$ was poured as a test solution. The $\mathrm{pH}$ of the solution was about 6. This solution was bubbled with $\mathrm{Ar}$ gas for at least 24 hours prior to an experiment and the bubbling was still continued during the experiment. A plate of Pt of $1 \mathrm{~cm} \times 1 \mathrm{~cm}$ was used as a counter electrode. In addition, there was a Luggin-Haber capillary in the cell to measure

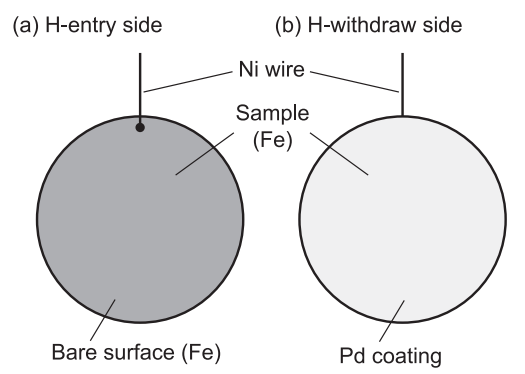

Fig. 1. Schematic drawing of the sample used for the electrochemical hydrogen-permeation tests.

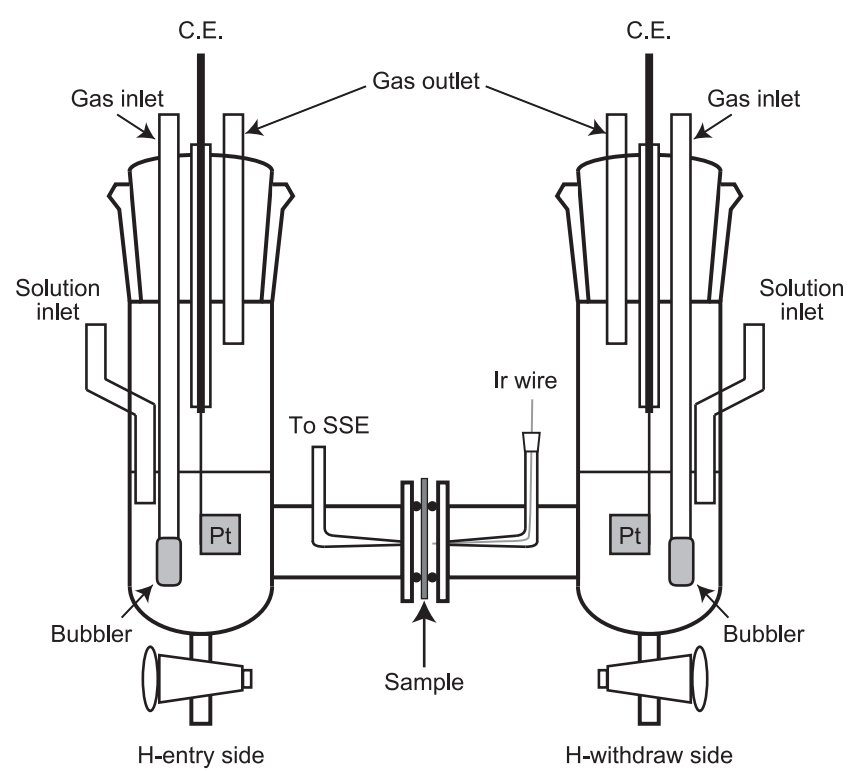

Fig. 2. Schematic drawings of the experimental setup for the electrochemical hydrogen permeation test. 
potential of the sample surface and the end of the capillary was connected to a reference electrode, $\mathrm{Ag} / \mathrm{AgCl}$ electrode in a saturated $\mathrm{KCl}$ (SSE).

On the other hand, an aqueous solution of $0.2 \mathrm{kmol} / \mathrm{m}^{3}$ $\mathrm{NaOH}$ was filled in $\mathrm{H}$-withdrawal-side cell. The solution was also bubbled with Ar gas continuously during an experiment. In this cell, the same shape of a Pt counter electrode as that in H-entry-side cell was used. However, in H-withdrawal-side cell, an Ir wire of $0.25 \mathrm{~mm}$ in diameter was employed as a reference electrode. ${ }^{18)}$

\subsection{Measurement of Hydrogen Permeation Current}

In this study, hydrogen absorption into an iron disk was detected with the change of oxidation current flowing out from the disk in H-withdrawal-side cell. The polarization potential of the disk facing to $\mathrm{H}$-withdrawal side was always $+0.1 \mathrm{~V}$ vs. Ir. At this potential, $\mathrm{H}$ atoms diffusing in the disk from H-entry side to H-withdrawal side can be oxidized completely to $\mathrm{H}^{+}$on the surface of the Pd coating. An HABF-501 potentiostat (Hokuto Denko Corp., Japan) was used to control the potential of the disk on $\mathrm{H}$-withdrawal side. Before pouring $0.5 \mathrm{kmol} / \mathrm{m}^{3} \mathrm{NaCl}$ solution in H-entry-side cell, the disk surface was kept at $0.1 \mathrm{~V}$ during the experiment.

On the other hand, $0.5 \mathrm{kmol} / \mathrm{m}^{3} \mathrm{NaCl}$ solution was poured in H-entry-side cell after the oxidation current in H-withdrawal-side cell reached almost a constant value at most as small as that in the order of several tens of $\mathrm{nA} / \mathrm{cm}^{2}$. Polarization potentials of a bare iron surface in H-entry side was varied in a step-up manner from $-1.2 \mathrm{~V}$ vs. SSE to open circuit potential (OCP). The potential was controlled with an HAL-3000 potentiostat (Hokuto Denko Corp., Japan).

In addition, in this study, sinusoidal waveforms of potential perturbation were superimposed to a polarization potential of iron disk in H-entry-side cell. The amplitude of the potential perturbation was a constant of $20 \mathrm{mV}$, which is a little larger than that normally used in the case of the measurements of electrochemical impedance spectroscopy (EIS). However, it was confirmed that current responses without any harmonic components were obtained against potential perturbation at each frequency. The perturbation frequency was varied in the range from $1 \mathrm{kHz}$ to $1 \mathrm{mHz}$ and swept from a higher frequency to a lower one successively in a logarithmic manner of 5 points per one decade. The measurement and analysis of the alternating responses of potential and current were performed with an FRA5014 4ch servo analyzer (NF Corp., Japan).

All the analog signal outputs from the potentiostats, potential and current, were measured with a Model 2700 digital multimeter equipped with a 20 ch multiplexer module (Keithley Instruments, Inc, US) and recorded in a personal computer with a home-made program written in Labview (National Instruments Corp., US).

\section{Results}

\subsection{Current Responses for Hydrogen Evolution and Absorption}

Figure 3 shows typical current responses of H-entry side, (b), and H-withdrawal side, (c), measured at various polarization potentials, (a). As mentioned in the experimental part, the polarization potentials are changed in a
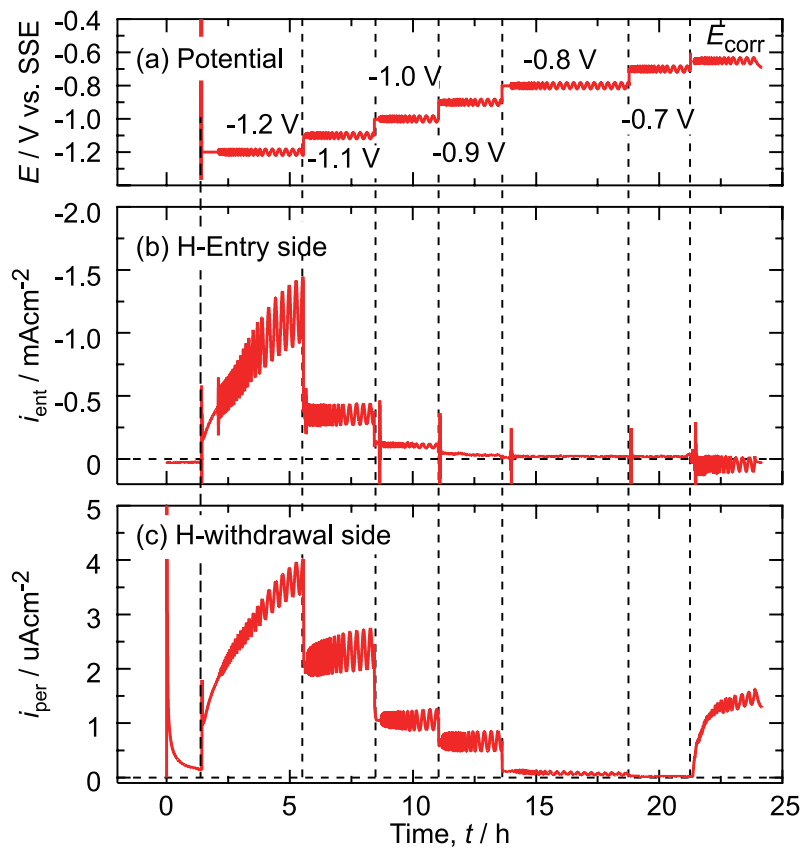

Fig. 3. Typical responses of polarization potential at an iron surface in H-entry-side cell, (a), current measured at H-entry side, (b), and current measured at H-withdrawal side, (c). (Online version in color.)

step-by-step from $-1.2 \mathrm{~V}$ to $E_{\text {corr }}$ and a small amplitude of potential perturbation of $20 \mathrm{mV}$ is superimposed to the polarization potentials. As shown in Fig. 3(b), the current responses for $\mathrm{H}$-entry side, $i_{\text {ent }}$ start to increase by applying cathodic potentials and reach almost stable levels after a certain time, although $i_{\text {ent }}$ is oscillated with the potential perturbation. The evolution of $i_{\text {ent }}$ is due to the acceleration of HER on iron surface by cathodic polarization. In addition, in Fig. 3(b), the average value of $i_{\text {ent }}$ at each polarization potential decreases as polarization potential becomes a more noble one.

In Fig. 3(c), current responses for $\mathrm{H}$-withdrawal side also start to increase by applying cathodic potentials and reach almost stable levels like the current responses for $\mathrm{H}$-entry side. The current responses for $\mathrm{H}$-withdrawal side are considered to be hydrogen permeation current due to oxidation of $\mathrm{H}$ atoms diffusing through an iron disk from the $\mathrm{H}$-entry side to the H-withdrawal side, meaning that hydrogen absorption into iron at the H-entry side. The hydrogen permeation current, here after $i_{\text {per }}$, is also oscillated with potential perturbation and as can be observed the magnitude of $i_{\text {per }}$ also decreases as the polarization potential becomes more noble potentials. These results indicate that HAR into iron can be enhanced with increasing cathodic polarization.

Figure 4 shows Bode plots of electrochemical impedance modulus and phase shifts measured at various polarization potentials. In Fig. 4, at the potentials from $-1.2 \mathrm{~V}$ to -0.9 $\mathrm{V}$, electrochemical impedance behavior can be expressed by an electric equivalent circuit with one time constant, namely simple Randles circuit. This indicates that cathodic reaction taking place on an iron surface in the potential region from $-1.2 \mathrm{~V}$ to $-0.9 \mathrm{~V}$ can be considered dominantly to be HER and that charge transfer resistance for HER increases with decreasing a cathodic polarization. In addition, the result of the electrochemical impedance behavior is in good agreement with that observed for $i_{\text {ent }}$ in Fig. 3(b). However, at the 


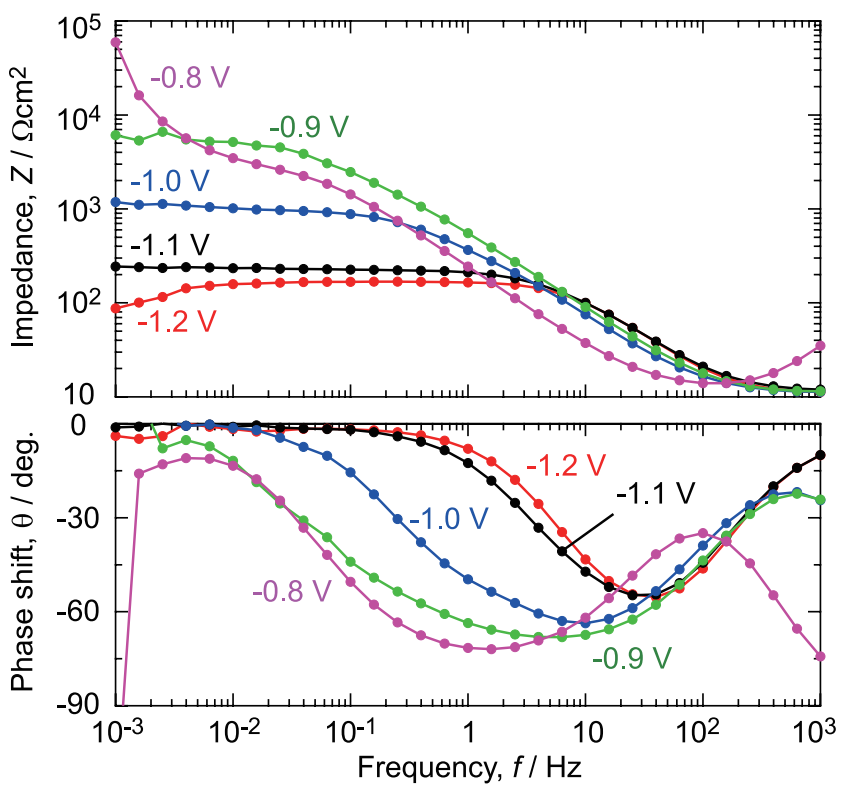

Fig. 4. Bode plots of electrochemical impedance spectra for iron measured in $0.5 \mathrm{kmol} / \mathrm{m}^{3} \mathrm{NaCl}$ with different polarization potentials. (Online version in color.)

potential of $-0.8 \mathrm{~V}$, electrochemical impedance behavior seems to be altered by comparison with that observed at less noble potentials than $-0.9 \mathrm{~V}$. This may be because the rate of HER becomes sluggish and the contribution of reduction reaction of oxygen dissolving with a very low concentration in a test solution to the total current is not negligible in addition to the effect of iron dissolution even though the test solution is being deaerated by Ar bubbling.

As observed in Figs. 3 and 4, the magnitudes of HER and HAR depend on the polarization potentials and they increase with increasing cathodic polarization. Here, in order to quantify the magnitudes of HER and HAR, the averaged values of $i_{\text {ent }}$ and $i_{\text {per }}$ measured at each potential, which are DC components of the current densities, are plotted as a function of polarization potentials. As shown in Fig. 5, the logarithms of the averaged values of $i_{\text {ent }}$ and $i_{\text {per }}$ decrease linearly as polarization potentials become more noble ones and Tafel slopes for both current densities can be evaluated to be $-170 \mathrm{mV} / \mathrm{dec}$ and $-270 \mathrm{mV} / \mathrm{dec}$, respectively. From the results of Tafel slopes for the averaged values of $i_{\text {ent }}$ and $i_{\text {per, }}$, reaction kinetics for HER and HAR can be analyzed. The reaction kinetics will be discussed in detail later.

Until this point, the DC components of $i_{\text {ent }}$ and $i_{\text {per }}$ in Figs. 3(b) and 3(c) have been focused on. In this study, since the oscillated current responses for $i_{\text {ent }}$ and $i_{\text {per }}$ have been obtained, the amplitudes of them, which are both $\mathrm{AC}$ components, can also be analyzed. By using the $\mathrm{AC}$ and DC components of $i_{\text {ent }}$ and $i_{\text {per }}$, hydrogen absorption efficiency, can be calculated in a different way by two methods. Namely, $i_{\text {per }} / i_{\text {ent }}$ and $\Delta i_{\text {per }} / \Delta i_{\text {ent }}$, can be obtained by dividing DC or AC component of $i_{\text {per }}$ by that of $i_{\text {ent }}$. Figure 6 shows potential dependency of hydrogen absorption efficiencies calculated by the above two procedures. The amplitudes of the current responses measured at $1 \mathrm{mHz}$ are being used as AC components for $i_{\text {ent }}$ and $i_{\text {per. }}$ As shown in Fig. 6, logarithms of hydrogen permeation efficiencies obtained with both AC and DC components increase linearly with decreasing cathodic polarization. This result means that the

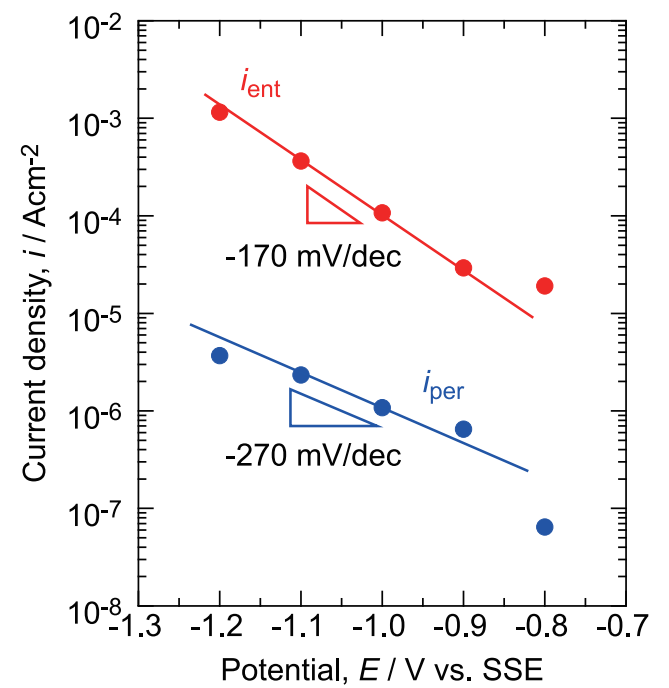

Fig. 5. Changes in the average values of $i_{\text {ent }}$ and $i_{\text {per }}$ as a function of polarization potential. (Online version in color.)

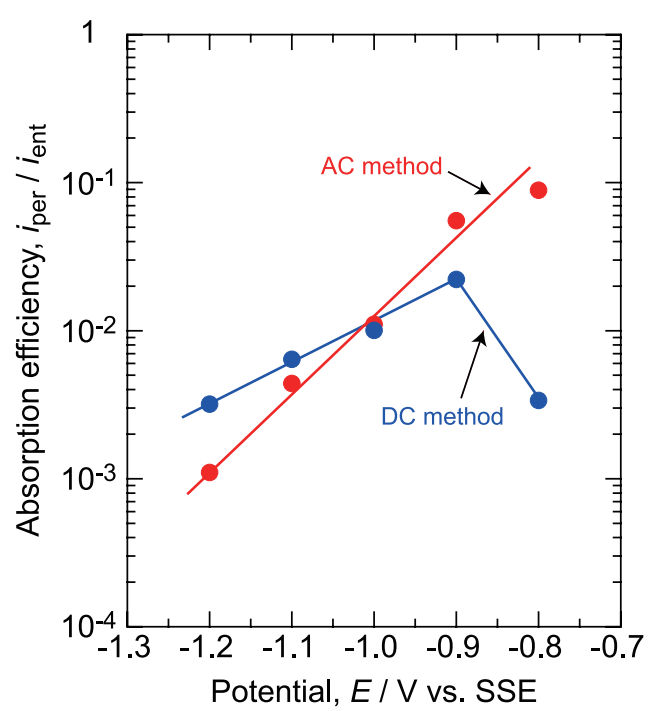

Fig. 6. Hydrogen absorption efficiencies for iron in $0.5 \mathrm{kmol} / \mathrm{m}^{3}$ $\mathrm{NaCl}$ as a function of polarization potential. (Online version in color.)

fraction of absorbed $\mathrm{H}$ atoms into iron against total amount of hydrogen evolved on an iron surface, namely hydrogen absorption efficiency, increases with decreasing cathodic polarization. In addition, it was elucidated that the efficiency is estimated to be 0.1 to $10 \%$. This suggests that most of the evolved $\mathrm{H}$ atoms by HER change into molecular $\mathrm{H}_{2}$ and some of the $\mathrm{H}$ atoms are absorbed into iron.

In addition, it is noteworthy that as shown in Fig. 6, there is a difference in potential dependency of hydrogen absorption efficiencies obtained by the AC and DC methods. In the case of the AC method, a liner relationship of the logarithm of the hydrogen absorption efficiency against polarization potential can be obtained up to $-0.8 \mathrm{~V}$. On the other hand, the efficiency obtained by the DC method decreases abruptly at $-0.8 \mathrm{~V}$ and the slope is slightly smaller than that by the AC method. These results may be related to the difference in the estimation of the current components of $\mathrm{AC}$ and DC for HER. In the DC method, the estimated current component can be affected by HER and oxygen reduction reaction (ORR). This means that the contribution of the cur- 
rent components due to ORR to the total current becomes larger as the cathodic polarization is decreased because HER slows down with decreasing cathodic polarization. On the other hand, in the AC method, the current component is considered to be directly related to the change in the magnitude of HER, because ORR is under a limiting current condition in the potential region employed in this study and can be dead to the potential perturbation. This suggests that the AC method is more preferable to evaluate hydrogen absorption efficiency than the DC method.

3.2. Frequency Response Characteristics of $i_{\text {ent }}$ and $i_{\text {per }}$

Figure 7 shows typical current responses of $i_{\text {ent }}$ and $i_{\text {per }}$ measured at $-1.0 \mathrm{~V}$. As shown in Fig. 7, after the potential perturbation with higher frequencies is applied, the oscillated current response of $i_{\text {ent }}$ with a large amplitude in the order of more than $100 \mathrm{uA} / \mathrm{cm}^{2}$ can be observed. By contrast, at this time, $i_{\text {per }}$ measured at higher frequencies is almost stable and does not include any oscillated responses. With decreasing perturbation frequencies of applied potential, the amplitude of $i_{\text {ent }}$ decreases and then reaches almost a constant at the lower frequencies than the order of several $\mathrm{Hz}$. This change of the amplitude of $i_{\text {ent }}$ is in good agreement with the results of the electrochemical impedance modulus in Fig. 3, meaning that the current oscillations of $i_{\text {ent }}$ measured at the lower frequencies are under a steady response at the applied potential of $-1 \mathrm{~V}$. On the other hand, it can be also observed that $i_{\text {per }}$ starts to be oscillated and that the amplitude of the oscillated response of $i_{\text {per }}$ increases with decreasing perturbation frequency. The amplitude of the oscillated response of $i_{\text {per finally reaches almost constant at }}$ lower frequencies than several $\mathrm{mHz}$. From these results, it is found that the amplitude of the oscillated $i_{\text {per }}$ depends on the perturbation frequency of applied potential.

Furthermore, from the results of the current responses
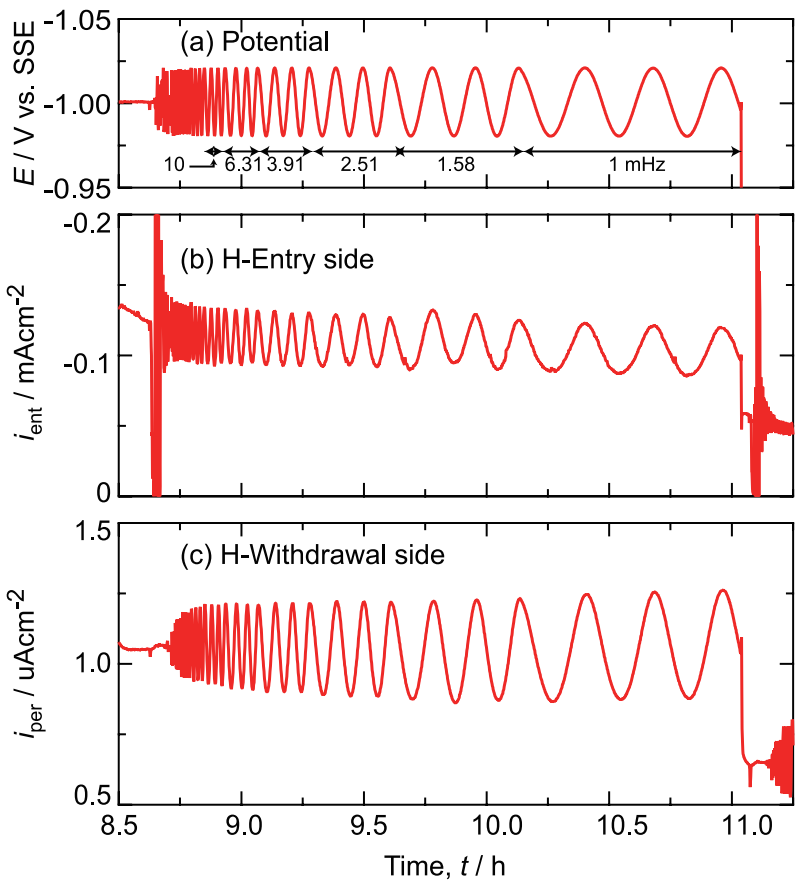

Fig. 7. Typical waveforms of currents for H-entry side and $\mathrm{H}$-withdrawal side measured at $-1.0 \mathrm{~V}(\mathrm{SSE})$ in $0.5 \mathrm{kmol} / \mathrm{m}^{3}$ $\mathrm{NaCl}$. (Online version in color.) of $i_{\text {ent }}$ and $i_{\text {per }}$ measured at various polarization potentials, frequency response characteristics of hydrogen absorption efficiency can be evaluated. In this study, the hydrogen absorption efficiency at each frequency has been calculated by using the data of electrochemical impedance modulus measured at H-entry side and $\mathrm{H}$-withdrawal side. The hydrogen absorption efficiency, $\Delta i_{\text {per }} / \Delta i_{\text {ent }}$ can be calculated by dividing electrochemical impedance modulus at H-entry side, $Z_{\text {ent }}$ by that at H-withdrawal side, $Z_{\text {per, }}$ as shown in Eq. (4).

$$
\frac{\Delta i_{\mathrm{per}}}{\Delta i_{\mathrm{ent}}}=\frac{Z_{\mathrm{ent}}}{Z_{\mathrm{per}}}
$$

Figure 8 shows frequency response characteristics of $\Delta i_{\text {per }} / \Delta i_{\text {ent }}$ evaluated at various polarization potentials. As shown in Fig. 8(a), $\Delta i_{\text {per }} / \Delta i_{\text {ent }}$ depends on perturbation frequency at each polarization potential and it decreases with increasing the frequency. At lower frequencies than about $10 \mathrm{mHz}, \Delta i_{\text {per }} / \Delta i_{\text {ent }}$ approaches almost constant values at all the polarization potentials used in this study. Therefore, in this study, $\Delta i_{\text {per }} / \Delta i_{\text {ent }}$ estimated at the lowest frequency of $1 \mathrm{mHz}$ is defined as the hydrogen absorption efficiency obtained by AC method. Furthermore, Fig. 8(b) shows phase shift between $i_{\text {ent }}$ and $i_{\text {per }}$ as a function of perturbation frequency. The changes in the phase shift measured at various polarization potentials are almost the same regardless of the polarization potentials and the phase shifts approaches to about $-180 \mathrm{deg}$. with decreasing perturbation frequency. The frequency response characteristics of $i_{\text {ent }}$ and $i_{\text {per }}$ will be discussed in detail in the following section.

\section{Discussion}

4.1. Potential Dependency of $i_{\text {ent }}$ and $i_{\text {per }}$

It was found from the results of Fig. 5 that the average

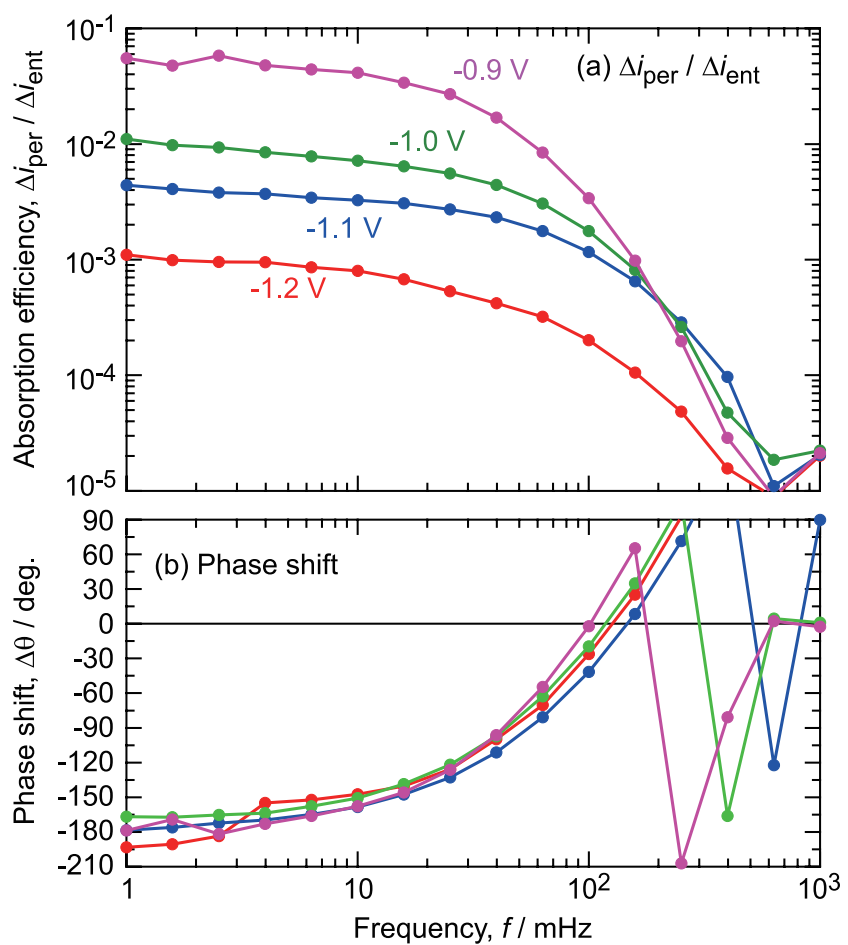

Fig. 8. Frequency response characteristics of hydrogen absorption efficiency for iron measured at various polarization potentials. (Online version in color.) 
values of $i_{\text {ent }}$ and $i_{\text {per }}$ obtained by DC and AC methods change linearly with the slopes of $-170 \mathrm{mV} / \mathrm{dec}$ and -270 $\mathrm{mV} / \mathrm{dec}$ as a function of polarization potential, respectively. Here, the potential dependency of $i_{\text {ent }}$ and $i_{\text {per }}$ will be discussed.

As explained in the introduction part, ${ }^{7)}$ HER on a metallic material proceeds step-by-step with Volmer reaction, Eq. (1), followed by Tafel reaction, Eq. (2) or Heyrovsky reaction, Eq. (3). $\mathrm{H}$ atoms are formed and adsorbed on the surface of the materials by Volmer reaction, and some of the adsorbed $\mathrm{H}$ atoms can be absorbed into the materials. Therefore, the evolutions of $i_{\text {ent }}$ and $i_{\text {per }}$ are associated with HER and HAR, Eq. (5) taking place on the surface of the materials, respectively.

$$
\mathrm{H}_{\mathrm{ad}} \longrightarrow \mathrm{H}_{\mathrm{ab}}
$$

Here, assuming that Volmer reaction is the ratedetermining step of HER on iron and that the following reaction is Tafel reaction, the expected slope for HER, $b_{\mathrm{c}}$ would be given by Eq. (6),

$$
b_{\mathrm{c}}=\frac{\partial E}{\partial \log i_{\mathrm{ent}}}=-2.303 \frac{R T}{\beta F} .
$$

where $E$ is electrode potential, $R$ the gas constant, $\beta$ symmetry factor for Volmer reaction, and $T$ the absolute temperature. When $T=298 \mathrm{~K}$ and $\beta=0.5, b_{\mathrm{c}}$ can be calculated to be $-120 \mathrm{mV} / \mathrm{dec}$, which is lower than that for the slope obtained in this study. Although there is a difference in the slope between the obtained value and the theoretical value, Volmer reaction is considered to be the rate-determining step for HER in this study. This is because if Tafel or Heyrovski reaction is assumed to be the rate-determining for HER, the Tafel slope is expected to be $-30 \mathrm{mV} / \mathrm{dec}$ or $-40 \mathrm{mV} / \mathrm{dec}$ at the same $T$ and $\beta,{ }^{7)}$ which is quite smaller by comparison with the slope of $-170 \mathrm{mV} / \mathrm{dec}$ obtained in this study. The reason why the obtained slope in this study is larger than that for the theoretical one of $-120 \mathrm{mV} / \mathrm{dec}$ may be the influence of ORR on the measurement of $i_{\text {ent }}$, as mentioned in section 3.1 .

Here, assuming again that Volmer reaction is the rate determining step of HER and the following reaction is Tafel reaction under an equilibrium state, the surface coverage of adsorbed $\mathrm{H}$ atoms on iron can be calculated as a function of electrode potential. ${ }^{19)}$ As reported in the literature, ${ }^{7,20,21)}$ the relationship between the surface coverage of adsorbed $\mathrm{H}$ atoms, $\theta$, and electrode potential, $E$, can be expressed by Eq. (7).

$$
\frac{\partial \log E}{\partial \log \theta}=2 \times\left(-2.303 \frac{R T}{\beta F}\right)=2 b_{\mathrm{c}}
$$

Furthermore, assuming that the reaction rate of hydrogen absorption in Eq. (5) is much faster than that of Volmer reaction, which means that $\mathrm{HAR}$ can be also regarded under an equilibrium, the surface coverage of adsorbed $\mathrm{H}$ atoms is proportional to $i_{\text {per }}$ when the diffusion of $\mathrm{H}$ atoms in iron is under a steady state and a limiting condition. This is because diffusion flux of $\mathrm{H}$ atoms in iron from $\mathrm{H}$-entry side to H-withdrawal side under a limiting condition obeys Fick's first law and the flux at the surface of H-withdrawal side reaches a constant. In this case, the slope of $i_{\text {per }}$ as a function of electrode potential would be the same as the value obtained by Eq. (7), resulting in $2 b_{\mathrm{c}}$. When $b_{\mathrm{c}}=-120$
$\mathrm{mV} / \mathrm{dec}$, the potential dependency of $i_{\text {per }}$ results in -240 $\mathrm{mV} /$ dec. As shown in Fig. 5, the slope of $i_{\text {per }}$ is $-270 \mathrm{mV} /$ $\mathrm{dec}$ and this value is quite similar to that of $-240 \mathrm{mV} / \mathrm{dec}$ theoretically obtained. Therefore, it can be considered from the results of Fig. 5 that HAR taking place at the H-entryside surface is faster than Volmer reaction.

\subsection{Frequency Response Characteristics of Hydrogen Absorption Efficiency}

As shown in Fig. 8, hydrogen absorption efficiency, $\Delta i_{\text {per }} / \Delta i_{\text {ent }}$ has frequency dependency at every polarization potential, showing it decreases with increasing perturbation frequency. Here the frequency response characteristics for $\Delta i_{\text {per }} / \Delta i_{\text {ent }}$ will be discussed.

As discussed in section 4.1, HER taking place on the $\mathrm{H}$-entry-side surface can be expressed by a rate-determining step of Volmer reaction followed by quasi-equilibrium Tafel reaction. In this case, $i_{\text {ent }}$ is proportional to square of the surface coverage of the adsorbed $\mathrm{H}$ atoms. ${ }^{7)}$ When HER is under a steady state at a given electrode potential, the surface coverage of the adsorbed $\mathrm{H}$ atoms is considered to be constant. This also means that the concentration of the adsorbed $\mathrm{H}$ atoms should be constant at a given electrode potential. Therefore, at a given electrode potential with a perturbation like in this study, the amplitude of $i_{\text {ent }}$ can be considered to be constant at the lower frequencies of potential perturbation less than $100 \mathrm{mHz}$.

On the other hand, as discussed in section 4.1, HAR taking place on the H-entry-side surface can be considered to be a faster reaction than Volmer reaction and then it can be regarded as an equilibrium state. In this case, $i_{\text {per }}$ can be considered to be proportional to the flux of $\mathrm{H}$ atoms at $\mathrm{H}$-withdrawal side. Therefore, in order to investigate frequency response characteristics of hydrogen absorption efficiency, $\Delta i_{\text {per }} / \Delta i_{\text {en }}$, frequency response characteristics of $i_{\text {per }}$ should be simulated.

First, one-dimensional profile of concentration of the absorbed $\mathrm{H}$ atoms in an iron membrane, $C_{\mathrm{H}}(x, t)$ can be expressed by Fick's second law, Eq. $(8)^{22,23)}$ under a boundary conditions given by Eq. (9):

$$
\frac{\partial C_{\mathrm{H}}(x, t)}{\partial t}=D \frac{\partial^{2} C_{\mathrm{H}}(x, t)}{\partial x^{2}}
$$

$C_{\mathrm{H}}(0, t)=C_{\mathrm{H}}^{0}+\Delta C_{\mathrm{H}} \sin (2 \pi f t), C_{\mathrm{H}}(L, t)=0$

where $L$ is the thickness of the iron membrane, $f$ the perturbation frequency, $t$ the elapsed time after applying potential, $x$ distance from $\mathrm{H}$-entry-side surface, $C_{\mathrm{H}}{ }^{0}$ the surface concentration of the adsorbed $\mathrm{H}$ atoms at $\mathrm{H}$-entry side at a given potential, and $\Delta C_{\mathrm{H}}$ the amplitude of the surface concentration of the adsorbed $\mathrm{H}$ atoms at $\mathrm{H}$-entry-side surface.

In this simulation, by assuming the concentration of the absorbed $\mathrm{H}$ atoms at $\mathrm{H}$-entry-side surface is defined as a function of perturbation frequency by Eq. (9), the fluxes of $\mathrm{H}$ atoms at $\mathrm{H}$-withdrawal-side surface can be calculated as a function of perturbation frequency by solving Eq. (8) with a COMSOL Multiphysics ver. 5.0 software (COMSOL Inc., US). At this time, we employ preliminary values for $C_{\mathrm{H}}{ }^{0}$ and $\Delta \mathrm{C}_{\mathrm{H}}$ as $1.0 \mathrm{kmol} / \mathrm{m}^{3}$, and $0.1 \mathrm{kmol} / \mathrm{m}^{3}$, respectively. In addition, diffusion coefficient of $\mathrm{H}$ atoms in iron used in this study was assumed to be $3.3 \times 10^{-9} \mathrm{~m}^{2} / \mathrm{s}$. Figure 9 shows 
the simulated results for the fluxes of $\mathrm{H}$ atoms at H-entryside surface $(x=0)$ and H-withdrawal-side surface $(x=L)$ as a function of perturbation frequency. As shown in Fig. 9, steady-state responses of oscillating $\mathrm{H}$-fluxes are observed in the later stage of the simulated results at all the perturbation frequencies. From the results of the H-fluxes, hydrogen absorption efficiency can be calculated as shown in Fig. 10. In Fig. 10, the frequency response characteristics of hydrogen absorption efficiency shows almost the similar trend against perturbation frequency. In addition, as shown in Fig. 9, the phase shifts between H-fluxes for H-entry side and $\mathrm{H}$-withdrawal side approaches 0 deg with decreasing perturbation frequency. These results indicate that the frequency response characteristics for hydrogen absorption efficiency observed in Fig. 8 is simulated very well with the assumed reaction scheme as mentioned above. From the results of this calculation, it is confirmed that HAR is a faster reaction than Volmer reaction and under a steady state.
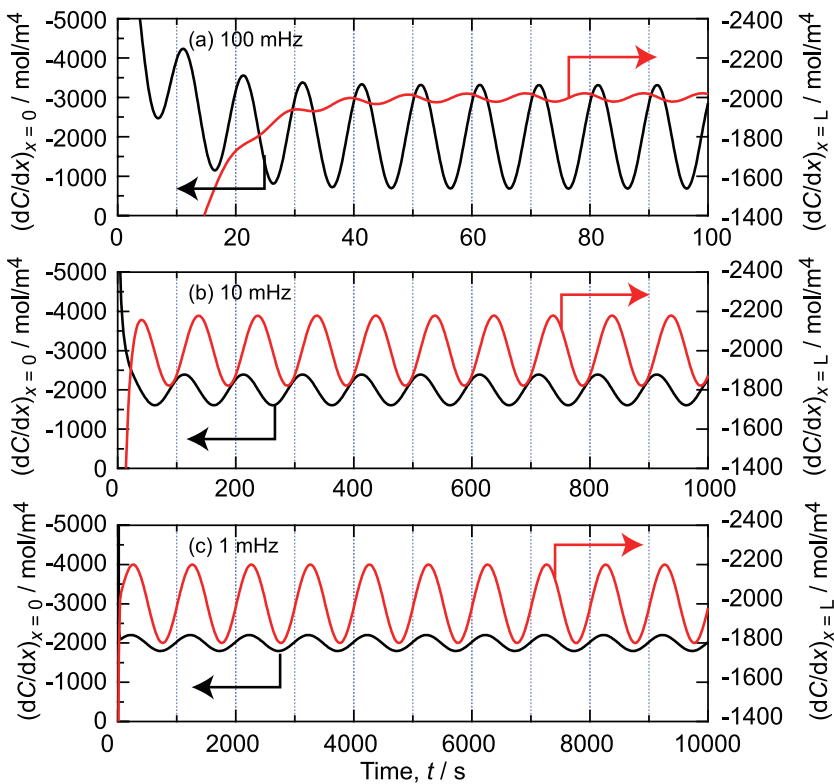

Fig. 9. Simulation results of hydrogen fluxes at both surfaces of $\mathrm{H}$-entry side and $\mathrm{H}$-withdrawal side at various perturbation frequencies. (Online version in color.)

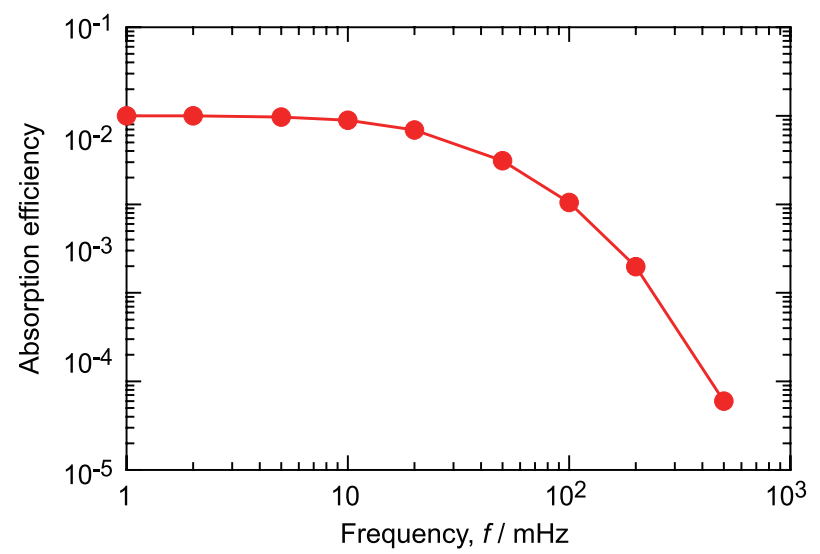

Fig. 10. Simulated frequency response characteristics of hydrogen absorption efficiency. (Online version in color.)

\section{Conclusion}

Hydrogen absorption behavior of iron in $0.5 \mathrm{kmol} / \mathrm{m}^{3}$ $\mathrm{NaCl}$ was investigated by analyzing alternation current responses in an electrochemical hydrogen permeation cell. The followings are the findings obtained in this study.

(1) Hydrogen absorption efficiency of iron was obtained successfully as a function of electrode potential. Hydrogen absorption efficiency tends to increase as polarization potential becomes more noble one.

(2) Hydrogen absorption efficiency of iron depends on the alternating frequency of polarization. The hydrogen absorption kinetics is considered to be faster than that of Volmer reaction and to be under an equilibrium state.

(3) It was elucidated that frequency response characteristics for hydrogen permeation current can be expressed by diffusion of $\mathrm{H}$ atoms in iron.

(4) It can be clarified that the measurements of alternating current responses is a very effective method for analyzing hydrogen absorption behavior at metal surfaces.

\section{Acknowledgement}

This work was performed as a part of the research "Comprehensive Understanding of Hydrogen-passive Surface on Steels for Prevention of Hydrogen Embrittlement" with a financial support by The Iron and Steel Institute of Japan. In addition, this work was also supported by JSPS KAKENHI Grant Number 26289268.

\section{REFERENCES}

1) S. Ootsuka, S. Fujita, E. Tada, A. Nishikata and T. Tsuru: Corros. Sci., 98 (2015), 430.

2) E. Akiyama, K. Matsukado, M. Wang and K. Tsuzaki: Corros. Sci., 52 (2010), 2758.

3) S. Li, E. Akiyama, T. Shinohara, K. Matsuoka and W. Oshikawa: ISIJ Int., 53 (2013), 1062.

4) T. Tsuru, Y. Huang, M. R. Ali and A. Nishikata: Corros. Sci., 47 (2005), 2431.

5) T. Omura, T. Kushida, F. Nakasato, S. Watabe and I. Oyamada: Tetsu-to-Hagané, 91 (2005), 478.

6) S. Matsuyama: Okure Hakai (Delayed Fracture), Nikkan Kogyo Shimbun, Ltd., Tokyo, (1989), 70.

7) E. Tada and T. Tsuru: Bull. Iron Steel Inst. Jpn., 17 (2012), 699.

8) N. Amokrane, C. Gavrielli, E. Ostermann and H. Perrot: Electrochim. Acta, 53 (2007), 700.

9) E. Protopopoff and F. Marcus: Corrosion Mechanisms in Theory and Practice, Chap. 2, 2nd ed., ed. by F. Marcus, Marcel Dekker, New York, (2002), 105.

10) M. M. Makhlouf and R. D. Sisson, Jr.: Metall. Mater. Trans. A, 22A (1991), 1001.

11) M. A. V. Devanathan and Z. Stachurski: Proc. R. Soc. Lond., Ser. A, 270 (1962), 90.

12) M. A. V. Devanathan and Z. Stachurski: J. Electrochem. Soc., 110 (1963), 886.

13) M. A. V. Devanathan and Z. Stachurski: J. Electrochem. Soc., 111 (1964), 619.

14) W. Beck, J. O’M. Bockris, J. McBreen and L. Nanis: Proc. R. Soc. Lond., Ser. A, 290 (1965), 220.

15) J. R. Scully and P. J. Moran: J. Electrochem. Soc., 135 (1988), 1337.

16) H. M. Ha, J. Ai and J. R. Scully: Corrosion, 70 (2014), 166.

17) I. Flis-Kabulska, T. Zakroczymski and J. Flis: Electrochim. Acta, 52 (2007), 2966.

18) T. Tsuru: Zairyo-to-Kankyo, 63 (2014), 3.

19) J. O’M. Bockris, J. McBreen and L. Nanis: J. Electrochem. Soc., 112 (1965), 1025.

20) R. N. Iyer and H. W. Pickering: J. Electrochem. Soc., 136 (1989), 2463.

21) R. N. Iyer and H. W. Pickering: Scr. Metall., 22 (1988), 1988.

22) N. Boes and H. Zuchner: J. Less-Common Met., 49 (1976), 223.

23) J. McBreen, L. Nanis and W. Beck: J. Electrochem. Soc., 113 (1966), 1218 . 